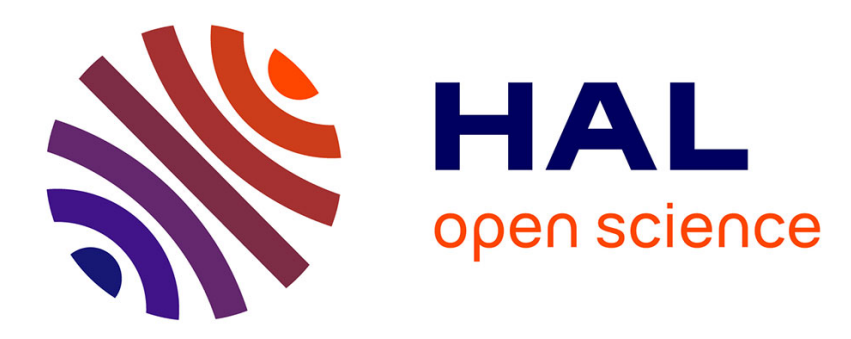

\title{
Gestion de l'eau et modèle de développement en débat en Espagne
}

Frédérique Blot

\section{To cite this version:}

Frédérique Blot. Gestion de l'eau et modèle de développement en débat en Espagne. Confluences, 2006, 58, pp.77-89. halshs-00912717

\section{HAL Id: halshs-00912717 https://shs.hal.science/halshs-00912717}

Submitted on 2 Dec 2013

HAL is a multi-disciplinary open access archive for the deposit and dissemination of scientific research documents, whether they are published or not. The documents may come from teaching and research institutions in France or abroad, or from public or private research centers.
L'archive ouverte pluridisciplinaire HAL, est destinée au dépôt et à la diffusion de documents scientifiques de niveau recherche, publiés ou non, émanant des établissements d'enseignement et de recherche français ou étrangers, des laboratoires publics ou privés. 
Frédérique Blot Toulouse II

Chercheure associée à l'UMR Dynamiques Rurales - Université

\title{
Gestion de l'eau et modèle de développement en débat en Espagne
}

\begin{abstract}
Les habitants du bassin du Segura, situé au Sud de l'Espagne, ont bien du mal à comprendre le Décret Royal du 18 juin 2004 qui pour eux constitue un retournement sans précédent au regard de la politique de gestion de l'eau menée en Espagne depuis plus d'un siècle. Cette évolution récente peut être attribuée à un débat régional : mais elle rend compte, aussi, de processus de régulation sociale liés à l'émergence des notions de "gestion intégrée" et de "gestion de la demande" qui interrogent simultanément modèles de développement et de gestion de l'eau.
\end{abstract}

A la suite d'un long conflit entre habitants du bassin de l'Ebre, mais aussi de querelles d'experts, le gouvernement espagnol a adopté le Décret Royal Loi du 18 juin $2004^{1}$ au lendemain du changement de majorité politique. Il a cependant été très mal accueilli dans le bassin du Segura (régions du sud-est de Valencia, de Murcia et d'Almería notamment). En effet, ce décret met fin aux projets de transferts d'eau prévus à partir du bassin de l'Ebre, au bénéfice de ce dernier. Or ces transferts étaient programmés par le récent Plan Hydrologique National (PHN) promulgué en juillet 2001.

L'Espagne est le pays européen le plus doté en infrastructures de régulation hydraulique ; les projets de transferts et barrages associés au PHN devaient pallier théoriquement les problèmes d'irrégularités de répartition spatiale et temporelle entre l'Espagne sèche et celle désignée comme excédentaire en eau. Le Décret Royal Loi du 18 juin 2004 apparaît d'autant plus surprenant que l'Etat espagnol avait présenté le PHN comme l'outil de planification qui permettrait de favoriser le développement économique sur l'ensemble du territoire national en résolvant le problème de l'eau.

En fait, ces représentations, qui lient développement et gestion de l'eau, accompagnent la politique de l'eau engagée en Espagne depuis le début du $\mathrm{xx}^{\mathrm{e}}$ siècle. Le retournement de juin 2004, qui apparaît de prime abord comme une mutation fondamentale, ne peut être interprété uniquement à l'aune de rapports de force régionalistes. Il convient donc d'explorer le modèle sous-tendu par le PHN afin de mieux 
saisir la complexité des processus de régulation et des conflits qu'il a suscités.

\section{Le PHN : la "gestion intégrale" de l'eau associée à un modèle de développement "productiviste"}

La mise en œuvre de la politique hydraulique nationale (conduite et coordonnée à l'échelle des principaux bassins-versants) menée en Espagne a pris la forme de projets globaux d'utilisation de l'eau depuis le Plan Gasset de 1902 jusqu'au PHN de 2001 ; celui-ci reprenait une seule des propositions du Plan proposé par Manuel Lorenzo Pardo en 1933 qui projetait la réalisation de grands transferts d'eau entre les différents bassins du pays. Il s'agissait alors de favoriser le développement agricole par l'accroissement des superficies irriguées.

Depuis, la politique de l'eau espagnole repose sur une "gestion intégrale" de toutes les sources potentielles en eau centralisée au niveau de l'Etat, c'est-à-dire sur une gestion quantitative planifiée visant à utiliser l'intégralité de celles-ci dans chaque bassin. L'eau est conçue en tant que matière renouvelable à l'infini, suivant un cycle qu'il convient de mettre à profit de façon intégrale (aprovechamiento integral de las aguas) avant qu'elle ne se perde en mer. Ainsi, l'efficacité de ce modèle de "gestion intégrale" se mesure au fait que les fleuves déversent un minimum d'eau en Méditerranée, comme l'exprimait Robert Hérin pour le fleuve Segura ${ }^{2}$.

Cette conception largement répandue a servi à justifier tous les aménagements visant à régulariser les flux d'eau dans le temps et à les répartir dans l'espace dans le contexte de ce que Michel Drain nomme un "paradigme hydraulicien" ou ce que Michel Marié nomme un «modèle aménagiste ${ }^{3}$. Les aménagements hydrauliques sont alors associés, dans l'imaginaire des populations, à l'idée de progrès social et économique pour lutter contre des conditions naturelles qui sont perçues comme des contraintes obligeant l'agriculture à faire face tantôt aux excès, tantôt aux pénuries en eau des fleuves.

Le bassin du Segura fournit une illustration toute particulière de ces représentations et pratiques de gestion. Considéré comme l'un des bassins les plus déficitaires de la péninsule ibérique, il est un véritable laboratoire de la mise en œuvre de la gestion intégrale. Manuel Lorenzo Pardo insistait dans le Plan de 1933 sur la nécessité de réguler les volumes «excédentaires» de l'un des bassins méditerranéens qui «offrent aux cultures irriguées leur environnement le plus propice ${ }^{4}$. La capacité de régulation hydraulique (capacité cumulée des barrages construits) a été ainsi multipliée par plus de 30 au cours du siècle. Lorsque la capacité interne de régulation annuelle du bassin de la Segura a été atteinte par la construction de barrages ${ }^{5}$, la solution préconisée dans le plan de 1933 fut envisagée pour soutenir la demande par l'offre: un transfert d'eau en provenance du Tage, l'Acueducto Tajo-Segura (ATS). Un canal de 292 kilomètres a été ainsi construit 
entre les bassins du Tage, du Jucar et du Segura et mis en service au cours des années 1978 et 1979.

Il faut rappeler qu'initialement, ces mesures se sont inscrites dans le cadre de la mise en œuvre du projet de développement autarcique d'une dictature, c'est pourquoi il n'y pas eu apparemment d'opposition politique à sa réalisation. C'est à partir de $1939^{6}$, sous Franco, que l'Instituto Nacional de Colonizacion (INC), devenu en 1971 l'Instituto de Reforma Y Desarrollo Agrario (IRYDA), a eu pour objectif de favoriser l'émergence d'une agriculture plus intensive et rémunératrice, utilisant des techniques de production "modernes" dont les produits sont tout d'abord destinés à la consommation intérieure en ayant recours à des "plans de développement agricole" fondés sur l'irrigation. Il s'agit avant tout d'une politique de colonisation des terres sèches. Dans ce cadre, l'INC déclare des zones d'intérêt national où il achète des terres vendues sur le principe du volontariat et crée des villages de colonisation : les campos.

Pour l'ensemble des zones sélectionnées les plans de développement agricole sont accompagnés d'autorisations d'exploitation des aquifères (nappes souterraines) et de réalisation d'infrastructures hydrauliques. La loi du 21 avril $1949^{7}$ qui octroie à l'Etat le droit d'exproprier et la totale compétence en matière de colonisation, s'oriente concrètement vers la transformation en regadíos (terres irriguées), à un rythme qui s'accélère jusqu'en 1965. Elle précise que «la colonisation et mise en irrigation des grandes zones du territoire national susceptibles d'être transformées grâce aux grands ouvrages hydrauliques réalisés par l'Etat [...] doit assurer des bénéfices économiques et sociaux pour la Nation tout entière ${ }^{8}$.

A partir de 1959, l'Espagne rompt dans la pratique avec une «politique autarcique » et adopte le "modèle de développement du monde libéral » ce qui se traduit par « une mise en valeur plus intensive des possibilités du pays ${ }^{9}$. En effet, la crise économique des années 1960 (inflation forte, émigration de la main-d'œuvre vers le reste de l'Europe, stagnation de l'industrie) pousse l'Etat, sur les conseils de l'OECE (Organisation Européenne de Coopération Economique) et de la BIRD (Banque Internationale pour la Reconstruction et le Développement) à adopter une politique économique libérale ${ }^{10}$. Dans ce cadre les cultures irriguées sont destinées à l'exportation. Ainsi «la règle entrepreneuriale, le critère de rationalisation et de modernisation économique, la norme du fonctionnement industriel $\gg^{11}$ gagnent du terrain. C'est dans ce contexte, l'Etat espagnol se fixe pour objectif la transformation de sa paysannerie; il s'agit de créer de véritables entreprises agricoles fonctionnant suivant «les règles $d u$ marché » : c'est-à-dire se fixant des objectifs de production élevés pour répondre à une demande intérieure en aliments, mais aussi pour susciter une demande extérieure.

La modernisation agricole passe alors par l'intensification des productions favorisées par la mécanisation, le recours aux intrants chimiques et le remembrement. Bien évidemment, un des leviers privilégiés de cette intensification réside dans la mise en irrigation de 
terres jusqu'ici non irriguées. En effet, "l'eau constitue le principal facteur en tonnage qui intervient dans la production agricole $\gg^{12}$.

Le Plan de desarrollo economico y social approuvé en 1963 et mis en œuvre à partir de 1964 s'inscrit clairement dans un modèle de développement "productiviste". En effet, ce dernier affiche alors pour "objectif primordial [...] de produire aux meilleures conditions de qualité et de prix le plus grand volume possible de biens et services ${ }^{13}$. Cela s'est traduit notamment par l'orientation des productions dans le Levant au cours des quarante dernières années: diminution de la production de blé et augmentation de la production d'agrumes puis de légumes primeurs destinés à l'exportation. Cette orientation a provoqué l'exploitation intensive des nappes dans les campos par les particuliers ou des sociétés privées destinées à l'exportation. Ils ont été tout d'abord les laboratoires de la modernisation agricole en Espagne et sont ainsi devenus aujourd'hui les modèles à suivre.

Parallèlement à l'agriculture ${ }^{14}$, l'ensemble des usagers de l'eau a été invité dès 1933 à en consommer davantage afin d'assurer un confort domestique et d'offrir des services touristiques, mais aussi de favoriser les productions industrielles puisque l'eau ne devait plus constituer un facteur qui peut les limiter. L'objectif central a été de fournir de l'eau en abondance à bon marché en libre service.

La prégnance de ce discours jusqu'à nos jours permet de comprendre pourquoi aujourd'hui dans le bassin du Segura les agriculteurs, les usagers domestiques, les usagers industriels ou les entreprises touristiques rencontrés dans le cadre de mes recherches ${ }^{15}$ comptaient encore sur des transferts d'eau pour satisfaire leur demande croissante et ne pas limiter le développement productiviste engagé jusqu'ici. Or les transferts prévus par la Ley del PHN de 2001 ont engendré un conflit virulent à propos des choix politiques de gestion des ressources en eau en Espagne qui ont entre autres conduit à la promulgation du Décret du 18 juin 2004.

\section{D'un conflit régional à une question de développement}

Dans les discours recueillis dans le bassin du Segura, les usagers et gestionnaires rencontrés dénoncent un conflit régional : une opposition Nord/Sud entre des communautés autonomes nanties et d'autres qui sont pauvres, tant en termes économiques et financiers qu'en dotation en eau. Le Décret de juin 2004 aurait été adopté sous la pression des communautés autonomes puissantes du Nord.

L'étude effectuée par Sylvie Clarimont ${ }^{16}$ dans le bassin de l'Ebre a montré qu'il existe effectivement une dimension régionale à l'opposition au projet de transfert d'eau entre bassins-versants, qui s'est exprimée dès les dernières années de la dictature franquiste. Par exemple, la presse aragonaise mettait en évidence l'opposition au projet de l'Etat de transférer de l'eau de l'Ebre vers l'Est des Pyrénées pour résoudre le problème de déficit en eau de la région barcelonaise. II ne s'agissait pas alors de refuser l'aménagement hydraulique, mais de préserver les «intérêts locaux» par «l'utilisation sur place des ressources en eau, implicitement définies comme bases de tout 
développement socio-économique, ressources en eau sur lesquelles les populations du bassin de l'Ebre auraient une sorte de droit de préemption $\gg{ }^{17}$. A l'époque il n'était pas encore question de remettre en cause la politique hydraulique associée à la création de barrages et de transferts en raison de leurs impacts écologiques et sociaux. Les implications environnementales en particuliers n'étaient pas considérées, et la mise en œuvre d'une "politique de l'offre" en eau fondée sur de grands aménagements structurants (grands barrages et transferts) était directement associée à l'idée de développement, soit à une croissance mesurée par des indicateurs essentiellement économiques.

Or le Décret de juin 2004 met fin au modèle de gestion intégrale de l'eau qui se réduit à une politique de l'offre. Il en souligne les lacunes des évaluations économiques (projets trop coûteux au regard des avantages produits), écologiques (impacts sur le delta de l'Ebre...) et techniques (faiblesse relative des quantités d'eau disponibles à long terme) qui ne permettraient pas d'obtenir les financements européens (FEDER) nécessaires à leur réalisation. Il signale aussi que la Ley del PHN présente des contradictions avec la Directive Cadre sur l'Eau européenne, et donc avec la législation espagnole qui la retranscrit tout particulièrement sur les impacts écologiques de la gestion de l'eau.

L'opposition au projet des transferts n'est par ailleurs ni unitaire, ni univoque. C'est pourquoi les dissensions régionales ne suffisent pas à expliquer un tel retournement. En fait, comme nous allons le voir, les processus qui ont donné lieu à la publication du Décret du 18 juin 2004 renvoient tout à la fois à des cadres de régulation sociaux (comme les relations conflictuelles) où sont mobilisés des arguments écologiques, économiques et sociaux, et à des cadres de régulation institutionnels (comme les directives européennes) où les principes de gestion adoptés sont censés fixer une nouvelle orientation à la politique de l'eau espagnole.

Dans le bassins de l'Ebre où l'eau devait être prélevée, ce n'est qu'à la fin des années 1980 que des considérations environnementales ont été mobilisées très distinctement en tant qu'arguments complémentaires pour refuser les transferts d'eau entre bassins, notamment par la "Coordination anti-transfert " (Coordinadora Antitrasvasament) qui associait les critiques d'un récent mouvement écologiste et d'élus locaux, d'agriculteurs mais plus généralement d'habitants du bassin de l'Ebre. Ce mouvement connut un nouvel élan lors de la proposition de l'avant-projet de PHN en 1993 : formation de la COAGRET (Coordination des Affectés par de Grands barrages et Transferts - Coordinadora de Afectados por Grandes Embalses y Trasvases), qui regroupe la Coordination anti-transfert et tous les collectifs de personnes affectées par de grandes infrastructures hydrauliques, ainsi que des associations écologistes influentes comme Greenpeace et la CODA (Coordination des Organisations de Défense Environnementale - Coordinadora de Organizaciones de Defensa Ambiental). 
Ce regroupement a donné lieu à la formulation de la notion de Nouvelle Culture de l'Eau (NCE). Il est appuyé depuis 1996 par de nombreux chercheurs qui ont organisé les Congrès Ibériques de Gestion et de Planification des Eaux. Des manifestations de grande ampleur ont été organisées après l'adoption du PHN comme la marche bleue au nom du "développement durable" pour une Nouvelle Culture de l'eau. Partie de Catalogne, en passant par la Vallée de l'Ebre, puis par la France elle rejoint Bruxelles en septembre 2001 pour interpeller la communauté européenne. Les manifestants critiquent alors une politique de l'eau qui, au nom du développement agricole, favorise uniquement une agriculture intensive polluante, et surtout la multiplication de services touristiques consommateurs en eau comme les terrains de golf et incite un processus de spéculation incontrôlé urbano-touristique.

Même dans le bassin du Segura, qui est supposé profiter du transfert, des conflits internes reprennent une partie des arguments environnementaux. Les communes situées à l'aval mènent un combat important au travers du collectif Pour un fleuve vivant, contre les prélèvements et les rejets polluants à l'amont du bassin. Les rivalités traditionnelles liées à la position des usagers au sein du bassin (amont/aval, rive gauche/rive droite) ont bien entendu toujours existé, mais depuis le milieu des années 1990 elles mobilisent aussi ces nouveaux arguments écologiques et sanitaires ; ceux-ci remettent en cause, de fait, le modèle productiviste adopté et favorisé par les politiques agricoles et industrielles. Du point de vue des riverains du fleuve, le Segura est un véritable "égout à ciel ouvert" dont l'aspect et l'odeur pestilentielle inspirent des craintes en termes de santé publique. Les membres de l'association Pro-Rio nous ont signalé que des familles riveraines du fleuve évoquent des cas de leucémie qu'ils attribuent à la mauvaise qualité de l'eau et de l'air.

Les problèmes de quantité d'eau disponible ont en effet un impact direct sur la qualité de l'eau puisque cette dernière découle des débits dans les cours d'eau; elle dépend en effet des prélèvements qui diminuent le taux de dilution et des rejets qui apportent des éléments divers (matières organiques, phosphates, composés azotés, métaux lourds, pesticides...).

Ainsi, toute la partie aval du bassin du Segura présente une qualité de l'eau considérée comme inadmissible, c'est-à-dire qu'il est impossible de produire de l'eau potable à partir de ces eaux même après traitement. La détérioration continue mesurée depuis 1972 est liée tout autant aux prélèvements agricoles importants qu'aux rejets non traités ou mal traités ${ }^{18}$ des industries et agglomérations réparties dans le bassin. Les nappes souterraines sont, elles aussi, affectées par des altérations qualitatives liées aux pollutions diffuses d'origine agricole, tout particulièrement par des nitrates et des pesticides ${ }^{19}$.

Les arguments écologiques et sanitaires mobilisés par les opposants aux transferts dans le bassin de l'Ebre et par quelques habitants et écologistes du bassin du Segura se font donc écho; en plus, ils s'appuient sur les discours émis au niveau d'instances internationales 
comme les Nations-Unies ou l'Union européenne, à l'image du Décret de juin 2004. Le PNUE' souligne par exemple les limites de "politiques de l'offre" en eau qui privilégient les grands aménagements particulièrement des barrages et transferts ; il les rend responsables de l'augmentation importante des prélèvements agricoles ${ }^{20}$. Quant aux expertises financées par l'Union européenne ou par le PNUE, comme celles réalisées dans le cadre du Plan Bleu, elles soulignent aussi les limites de ces politiques ${ }^{21}$.

Le cas du bassin du Segura qui a déjà fait l'objet d'un transfert en provenance du Tage illustre concrètement le caractère non durable de ces politiques. En effet, les volumes transférés en provenance de ce fleuve depuis 1979 (projet ATS, Acueducto Tajo-Segura) n'ont jamais atteint les attentes initiales en raison du caractère aléatoire des précipitations et de l'augmentation des prélèvements dans le bassin dit excédentaire. Les agriculteurs qui dépendent de cette ressource ont donc été contraints de mobiliser leurs autres sources d'eau. Agriculteurs "modernes", ils utilisent aujourd'hui en majorité la technique du goutte-à-goutte qui nécessite des apports réguliers et fréquents ce qui accroît la productivité et l'efficience de cette agriculture mais la rend aussi beaucoup plus vulnérable au stress hydrique. C'est pourquoi ces agriculteurs ont exploité de plus en plus massivement les nappes d'eau souterraines desquelles ils dépendent depuis la création des campos mais dont la capacité de renouvellement est inférieure aux prélèvements d'une agriculture intensive: les nappes sont ainsi surexploitées.

De plus, ce phénomène s'est accentué en raison de l'entrée en fonction de l'ATS dont la simple approbation en 1968 avait déjà provoqué des transformations de secanos (terres non irriguées) en regadíos: ce sont 57000 hectares de cultures irriguées en plus des 50880 prévus dans le projet de l'ATS qui ont été légalisés depuis cette approbation $^{22}$ dans la région de la Segura. Ce qui promet de se reproduire puisque les agriculteurs rencontrés ont précisé que selon eux l'arrivée de l'eau de l'Ebre devrait leur permettre d'accroître leurs prélèvements et de répondre ainsi à la demande en eau de leurs cultures existantes et de nouvelles cultures. La politique de l'offre en eau mise en œuvre ici peut donc être associée à un véritable cercle vicieux où l'offre provoque une demande croissante qui requiert une offre supplémentaire qui provoque à son tour une demande nouvelle... Ces politiques de l'offre visant à réduire les irrégularités de répartition spatiale et temporelle des flux d'eau annuels, conduisent de fait à gommer les particularités des régimes hydrologiques des cours d'eau : l'objectif est d'obtenir des volumes optimaux quasiment identiques tout au long de l'année. Certes, les irrégularités hydrologiques sont atténuées, mais les volumes diminuent en raison des prélèvements croissants dans l'ensemble du bassin. Malgré la mise en œuvre d'une "gestion intégrale" des eaux, figurée par la mise en service de

1 Programme des Nations-Unies pour l'Environnement, organe promoteur du "développement durable". 
nombreuses infrastructures de régulation, la demande en eau dépasse constamment et de façon croissante les disponibilités réelles du bassin. Le décalage entre apports réels en eau au bassin et demande s'est avéré croissant ce qui se traduit par la surexploitation des nappes, la diminution des débits du fleuve et est exprimé par la confédération hydrographique, gestionnaire institutionnel du fleuve, en termes de déficit croissant au regard de la demande en eau (de 272 à 460 millions de mètres cubes entre 1972 et $1998^{23}$ ).

Les diagnostics réalisés dans le cadre de la mise en œuvre de la Directive Cadre sur l'Eau confirment la prégnance de ces problèmes de gestion au regard des diagnostics effectués en fonction des objectifs de qualité écologique à atteindre pour 2015. Dans le bassin du Segura du point de vue quantitatif (débits ou volumes minimum admissibles dans les masses d'eau) $63 \%$ des masses d'eau souterraines ne pourront atteindre les objectifs fixés par la DCE; du point de vue qualitatif (critères physico-chimiques et écologiques) $71 \%$ ne pourront les atteindre. Mais lorsque l'on couple les indicateurs qualitatifs et quantitatifs ce sont $92 \%$ des masses d'eau souterraines qui ne pourront atteindre ces objectifs ${ }^{24}$. En ce qui concerne les eaux de surface, seule la partie amont du bassin (amont à l'arrivée de l'ATS) pourra atteindre cet objectif de bon état dans l'attente d'études complémentaires.

On le voit bien, la situation de déficit quantitatif et d'altération qualitative des sources d'eau du bassin du Segura va donc à l'encontre de l'opinion communément admise selon laquelle du point de vue technique, la politique de l'offre aurait dû permettre de résoudre ces problèmes puisque telle était la prétention de sa proposition. Cette situation est en ce sens a priori paradoxale mais s'explique parce que dans le contexte où la politique a été formulée toutes les interdépendances n'ont pas été prises en compte: interdépendances sociales au sein des régions concernées et interdépendances écologiques au sein des hydrosystèmes. En effet, l'éventualité de la transformation des pratiques n'a pas été envisagée. Par exemple dans le bassin du Tage et dans la province d'Albacete le maïs irrigué s'est développé et prélève à la fois des eaux du Tage (et donc diminue les débits potentiellement transférables par l'ATS) et des eaux dans les nappes qui approvisionnent le bassin du Segura. De même, l'augmentation importante de la demande en eau potable et de l'assainissement liés à l'accroissement du tourisme sur la côte n'a pas été intégrée à la réflexion. Les aléas de l'alimentation hydrologique pourtant caractéristiques du bassin du Segura de même que les relations entres nappes et eaux de surface n'ont pas non plus été considérés.

Or dans le mouvement d'ensemble récent associé à la notion de "développement durable" ces interdépendances devraient, suivant les principes de la "gestion intégrée" 25 , être prises en compte pour gérer les relations au sein des hydro-socio-systèmes considérés. Aussi convient-il de ne pas réduire les processus de régulation conflictuels à des conflits d'intérêts: plus globalement, ils rendent compte de l'évolution potentielle des relations entre sociétés et ressources, et entre sociétés autour de la Méditerranée, dans le contexte de la mise en 
œuvre d'un modèle plus durable. A l'heure de la promotion de politiques de gestion de la demande s'insérant dans des processus de gestion intégrée des ressources en eau, le modèle de gestion intégrale et le développement productiviste qui lui est associé paraissent donc remis en cause. C'est pourquoi l'exemple de la politique de l'eau menée en Espagne, tout particulièrement dans le bassin du Segura, constitue un cas à étudier et à méditer pour l'ensemble méditerranéen. 
Notes :

1 “ Real Decreto Ley 2/2004 de 18 de junio ”, BOE n¹48, 19 junio 2004.

2 HerIN Robert (1976), Le bassin du Segura, Thèse de doctorat de géographie Rurale, Université de Caen, p. 209.

3 MARIÉ Michel (2003), “ La formation d'un modèle aménagiste de l'eau ", dans DRAIN Michel [dir], Politiques de l'eau en milieu Méditerranéen. Le cas de la péninsule Ibérique, Madrid, Casa de Velázquez, Universidad de Alicante, p. 1527.

${ }^{4}$ Lorenzo Pardo Manuel (1933), “Tomo I”, Plan nacional de obras hidráulicas, Madrid, MOPU, p. 40.

${ }^{5}$ C'est-à-dire lorsque les retenues qui étaient susceptibles d'être remplies par les flux d'eau annuels dans le bassin ont été construites.

${ }^{6}$ Decreto de 18 octubre de 1939 « organizando el INC », BOE, 27 oct.1939.

7 " Ley sobre colonización y distribución de propiedad de las zonas regables ", Ley de 21 de abril 1949, BOE, $\mathrm{n}^{\circ} 112$, de 22 de abril de 1949.

8 Ministerio de agricultura, InStituto NaCIONAL de Colonización (1949), Ley sobre colonización y distribución de propiedad de las zonas regables, I.N.C Publicaciones, 1 a serie $-\mathrm{n}^{\circ} 12$, p. 3.

9 DaUmas Max (1980), Mélanges hispaniques, Toulouse, UTM, p. 55.

${ }^{10}$ L'Espagne s'est engagée auprès de l'OECE, du FMI et de la BIRD dès 1958.

11 OrTega Cantero Nicolás (1993), “ Algunas orientaciones de la política agraria española posterior a la guerra civil: de la colonización a la ordenación rural ", dans GIL OlCINA Antonio, Morales GIL Alfredo [Ed.] (1993), Medio siglo de cambios agrarios en Espana, Alicante, Instituto de cultura Juan Gil-Albert, p. 21.

12 NARedo Pérez José Manuel (1999), “Consideraciones econónomicas sobre el papel del agua en los sistemas agrarios ", dans GARRABOU SEGURA Ramon, Naredo Perez Jose Manuel [eds.] (1999), El agua en los sistemas agrarios. Una perspectiva historica, Madrid, Fundación Argentaria, VISOR, p. 65.

${ }^{13}$ RUdel Christian (1966), L'Espagne du Plan ou la succession ouverte, Paris, Les éditions ouvrières, p. 28.

${ }^{14}$ Le Plan de 1933 préconisait déjà de facilité l'accès à l'eau pour l'ensemble des usages.

15 BLOT Frédérique (2005), Discours et pratiques autour du "développement durable" et des "ressources en eau". Une approche relationnelle appliquée aux bassins d'Adour-Garonne et du Segura, thèse de doctorat, Toulouse, 543 p.

${ }^{16}$ Sylvie Clarimont, dont la thèse porte sur les conflits pour l'eau dans le bassin de l'Ebre a décrit de façon approfondie l'émergence du mouvement antitransfert en Aragon. (1999), Les conflits pour l'eau en Europe méditerranéenne : le cas du bassin de l'Ebre (Espagne), Thèse de doctorat, Montpellier, p. 233-286.

17 Clarimont Sylvie (1996), “Conflits pour l'eau dans le bassin de l'Ebre ”, dans Espace rural, $\mathrm{n}^{\circ} 36$, Montpellier, p. 103-104.

18 D'après le Plan Hydrologique du bassin du Segura, sur les 192 stations d'épuration du bassin, seules 81 fonctionnement correctement, 66 n'assurent pas l'assainissement suffisant des effluents qui y sont traités et 45 ne fonctionnent pas du tout. CHS - Confederación Hidrografica del Segura (2000a), Memoria completa del Plan Hidrologico de la cuenca del Segura, Murcia, Ministerio de Medio Ambiente, p. 247.

${ }_{19}$ CHS (2000b), “Anejo 5. Programación ", Plan Hidrologico de la cuenca del Segura, Murcia, Ministerio de Medio Ambiente, p. 185, 189.

20 PNUE (2002), L'avenir de l'environnement mondial 3. GEO-3, Paris, De Boeck Université s.a, p. 151. 
${ }^{21}$ Benolt Guillaume, Comeau Aline [dir.] (2005), Méditerranée. Les perspectives du Plan Bleu sur l'environnement et le développement, La Tour d'Aigues, L'Aube, p. 79.

${ }^{22}$ Martínez Fernández Julia, Esteve Selma Miguel Angel (2000), Sequía estructural y algunas externalidades ambientales en los regadíos de la cuenca del Segura, Ingeniería del Agua, vol. 7, $\mathrm{n}^{\circ} 2$, p. 59.

${ }^{23}$ CHS (2000c), "Orden del 13 de agosto de 1999 por la que se dispone la publicación de las determinaciones de contenido normativo del Plan Hidrológico de la cuenca del Segura, aprobado por el Real Decreto 1664/1998, de 24 de julio", Plan Hidrologico de la cuenca del Segura, Murcia, Ministerio de Medio Ambiente.

${ }^{24}$ CHS (2005), Informe resumen de los artículos 5, 6 y 7 de la DMA, Murcia, p. 11-12.

${ }^{25}$ Principes que je me suis attachée à étudier dans le cadre de ma thèse puisque la "gestion intégrée" est présentée comme un des outils pour mettre en œuvre un nouveau modèle de "développement durable". J'ai montré que théoriquement la gestion des ressources en eau devrait être appréhendée au sein d'hydro-socio-systèmes afin de prendre en compte l'ensemble des interactions en jeu, et de favoriser une gestion symétrique des points de vue sociaux et écologiques. 